EPiC Series in Language and Linguistics
Volume 3, 2018, Pages 53-58
LSP in Multi-disciplinary contexts of
Teaching and Research. Papers from the
16th International AELFE Conference

\title{
The proximal narratives of online risk communication: fatal aircraft-accident synopses by the U.S. National Transportation Safety Board
}

\author{
Carmen Sancho Guinda \\ Universidad Politécnica de Madrid, ETSI Aeronáutica y del Espacio, Plaza del Cardenal Cisneros \\ 3, 28040-Madrid, Spain \\ carmen.sguinda@upm.es
}

\begin{abstract}
I explore the narrative strategies adopted by a specific type of institutional discourse grounded in the authority of expertise: risk communication, aimed at influencing our conceptions of hazard and danger to ultimately prevent unsafe behaviours. My focus is the unique discourse of the fatal aviation accident synopses issued yearly online by the National Transportation Safety Board of the United States of America (NTSB for short). Through a blended framework that merges Narratology, Critical Discourse Analysis and the Positioning and Proximisation Theories, I examine how the NTSB didactically brings risks and dangers close to its broad mixed virtual audience while undertaking fluid roles for branding purposes and disseminating the ideological principles of American democracy. In doing so, I especially attend to issues of narrative focalisation (i.e. recounting perspective) and speech representation.
\end{abstract}

Keywords: Risk communication; proximisation; aircraft-accident dockets online; U.S. National Transportation Safety Board

\section{The Proximal Nature of Risk Communication}

Risk communication (henceforth RC) has been recently defined as an epistemic category (Boholm \& Corvellec, 2014) based on both rationality and emotion and culture-bound, intended to prevent unsafe behaviours and outcomes, detect deficiencies in safety systems, and ultimately recommend future courses of action. Communicating risk also relies on a tacit social contract that involves credibility, trust, and procedural fairness (Besley \& McComas, 2014), the latter ensuring audience participation, as well as on the construction of psychological distance (Zwickle \& Wilson, 2014), which aims at bringing the consequences of the danger or hazard at issue closer to the addressee in order to make them vivid and thus raise his/her awareness more sharply. Critical Discourse Analysis 
has profusely investigated the manipulation of 'deictic centres' in what Cap (2013) has termed proximisation theory, applied to the study of the legitimation of vested interests and biased policies. Through proximisation entities and events become less abstract and more concrete on the spatial, temporal, and social planes: 'then' becomes 'now', 'there' is turned into 'here', and the otherness of dissimilar social groups is substituted by nearness and similarity. For the sake of efficacy, then, RC is proximal by its very nature, since it leans on making individuals feel dangers as probable and immediate.

\subsection{Proximal Uniqueness of U.S. Fatal Air-accident Dockets Online}

In this paper I discuss the proximal discursive strategies of a singular type of RC: the dockets of fatal aviation accidents issued yearly online by the U.S. National Transportation Safety Board (NTSB for short) in its website. This proactive digital dissemination, which started on July 1, 2009, complies with (and simultaneously transmits) one of the tenets of North American democracy: the institutional transparency enacted by the FOIA (Freedom of Information Act, in force since 1967), whereby citizens are ensured trustworthy and accessible communication with governmental agencies. Being a digital-medium genre, the NTSB docket reaches a broad hybrid readership of experts and laypeople and display the format of an average conventional scientific abstract, with an extension that ranges from 100 to 400 words. According to the severity of the mishap aftermath, NTSB dockets are classified into fatal (causing deaths), non-fatal (causing injuries), and incidents (with just technical repercussions), and depending on the investigation stage, into preliminary (reporting still at an early phase), factual (providing data but not speculating on causes, mostly because a foreign government is in charge of the investigation), and of probable cause (speculative as to the causes of the accident and the ethics of the actors involved, normally the crew and maintenance personnel). The object of my study has been a compilation of fatal probable-cause dockets, under the criterion that they should employ richer discursive devices than the other two docket types to express their epistemic evaluation and subtle moral criticism. This hypothesis has been confirmed by the two major proximisation strategies found: multivocality (i.e. the inclusion of direct speech testimonies and quotations for different purposes), and in medias res storytelling (i.e. the absence, while reporting, of a narrative frame for actions, recounted as already in course in the middle of the plot).

The docket structure comprises several headings succinctly introducing the basic facts of the accident (see Example 1, my italics):

(1) NTSB Identification: CEN10LA274

14 CFR Part 91: General Aviation

Accident occurred Friday, May 28, 2010 in Granbury, TX

Probable Cause Approval Date: 04/28/2011

Aircraft: HENNEY MICHAEL A VANS RV-6A, registration: N226MH

Injuries: 1 Fatal,1 Serious.

NTSB investigators may not have traveled in support of this investigation and used data provided by various sources to prepare this aircraft accident report.

The pilot and passenger were on the final approach to their destination airport, after completing about a 550-nautical mile, cross-country flight in a kit-built airplane. During the approach, the left wing dropped and the airplane entered a steep descending bank before impacting the terrain. The surviving passenger could not recall details of the accident. A postaccident examination of the airframe and engine wreckage did not reveal any abnormalities that would have precluded normal operation.

The National Transportation Safety Board determines the probable cause(s) of this accident to be: 
The loss of control for undetermined reasons.

\section{$\underline{\text { Full narrative available }}$}

These headers are followed by the body of text, whose informative thread is formed by attribution (e.g. reporting verbs and evidential adverbials such as 'according to a witness' or 'Toxicological analysis revealed') and ends with a formally and performatively enunciated verdict, with a characteristic phraseology ('The National Transportation Safety Board determines the probable cause(s) of this accident as follows:...'). In (1) I have italicised this sort of attributive expressions, infallibly sequenced in a growing scale of objectivity: first the subjective testimonies by witnesses recalling the facts, then the findings and interpretations of experts analysing diverse types of evidence (wreckage remains, impact on the terrain, photos and films taken by witnesses, cabin voice recordings, toxicological and pathological tests, etc.), and finally the conclusion of the Board as closing verdict. Curiously enough, this formulaic verdict compactly imposes the Board's voice of authority by condensing all the previous testimonies and interpretations, which are shown to corroborate the institution's expertise and reliability. Multivocality is consequently in the service of univocality.

\subsection{Rationale behind the NTSB's Proximal Discursive Strategies}

We may wonder why the NTSB does not adopt a sanitized style focused on strict factual reporting, as do all other national agencies for transportation and safety round the world. The reason is simple: because of the legal framework set by the FOIA, added to the didactic quality and the goal of maximum dissemination inherent in most instances of $\mathrm{RC}$ and the popularisation of scientific and technical contents brought about by computer-mediated communication, which on the whole has made interactions more informal (Hewings, 2012; Zhang \& O'Halloran, 2014; Hyland \& Jiang, 2017). The transparency prescribed by the FOIA can be broken down into two main components: accessibility and informativeness. Making specialized content accessible means spreading it widely (the NTSB's option for the monthly publication of dockets in its webpage and its periodic upgrades meet this requisite) and conveying the message intelligibly, which in turn implies the conditions of explicitness and memorability. Being informative, for its part, presupposes a regulation of the level of disclosure (a sufficient and adequate amount of detail or granularity, as defined by van Dijk, 2016) and democratic reporting; that is, multivocality.

Hence, the 'transgressive' storytelling of NTSB fatal aircraft-accident dockets tries to achieve the aforementioned elements of communicative transparency: their drama features and the plurality of voices (e.g. witnesses, crew, survivors, experts, technical and legal literature), the emotional language and the level of detail, contribute to improving comprehension and memorising long-term routines by a mixed audience. In addition, detail and multivocality provide greater precision than a monovocal account from a single narrative perspective (i.e. from an omniscient narrator), so they impinge on the NTSB's credibility and openness as a participative governmental institution, and should therefore be regarded as tools for political branding.

\section{Method}

Essentially qualitative, this research draws on the manual examination of an electronic corpus of 512 NTSB docket samples online covering the time span 2010-2015, all of them belonging to the fatal and probable cause types and retrievable from the website www.ntsb.gov/ layouts/ntsb.aviation/month.aspx. 
My theoretical framework is an eclectic one merging Narratology, Critical Discourse Analysis and the Positioning (Harré \& van Langenhove 1999) and Proximisation Theories (Cap 2013, Zwickle \& Wilson, 2014). Each sample underwent the following procedure:

- Examination of narrative opening (whether conventional framing of actions or in medias res)

- Detection of direct speech reporting and interpretation of functions

- Analysis of remarkable detail use

\section{Findings: The Synergy of Multivocality and in medias res Storytelling}

Thorough visual inspection of the samples soon revealed the interest of multivocality (and its subsequent level of narrative detail) and in medias res storytelling as objects of study, because they seem to interact synergically to empower the audience by shortening psychological distance and to boost intelligibility, informativeness, and institutional ethos. Along Harré and van Langenhove's (1999) lines, I hold that, out of communicative efficiency and together with the expectable roles of technical expert and government representative, the NTSB undertakes those of knowledge and emotional mediator, and peer citizen. These multiple roles find their discursive translation as sensitivity towards the Board's heterogeneous audience, in the form of content glossing through climactic storytelling, direct speech quotations, and of rapport and intimacy building through a conversational, occasionally even gossipy tone, that may uncover private personal information (chiefly medical, penal and sentimental).

\begin{tabular}{ll}
\hline Proximal strategy used & \% samples \\
\hline In medias res narrative openings & 98.24 \\
Direct speech reporting & 21.48 \\
Singular use of detail & 10.74 \\
\hline
\end{tabular}

Table 1: Shortening of psychological distance in NTSB fatal air-accident dockets

Table 1 shows that in medias res narrative openings are overwhelmingly resorted to, presumably because the headers at the top of the docket document fulfill the framing function of a linear narrative. By contrast, direct speech reporting and singular uses of detail, which go hand in hand, are much less frequent, although their sole presence in this type of technical documents is already remarkable.

\subsection{In medias res Narrative Openings}

In medias res openings are, above all, attention-catchers that prepare the scene prior to the accident's denouement. They may refer to events previous to the accident (past reference) - for instance to actions performed by the crew hours, days, weeks or months before, to the reporting by witnesses and experts some time after the catastrophe, or to a span of time in which the mishap is about to happen (2).

(2) The commercial pilot departed a remote, oceanside lagoon in a float-equipped airplane with three passengers on an on-demand air taxi flight in reduced visibility and heavy rain. (NTSB Identification: ANC10FA100)

This last alternative is the most engaging one, due to its fictional 'thriller-like' quality. It creates an atmosphere of suspense that captures the addressees' interest and encourages them to read on. 


\subsection{Direct Speech Reporting}

Quotations play a triple function: Firstly, they simplify technical concepts by building didactic similes or supplying known schemata into which to categorise new phenomena. In this sense, the sui generis onomatopoeias and comparisons in witnesses' testimonies (3) are crucial to facilitate expert diagnoses. Secondly, quotations enhance the authority of verdicts through the verbatim transcription of normative literature (e.g. regulations, operation manuals, etc.) (4).Thirdly, they confer the account an emotional tone very helpful to evaluate the factors and effects of the risk in question (5). Italics are mine in all cases.

(3) A witness observed the helicopter depart and begin aerial spray operations in a corn field. He turned away and heard a loud "pop," then turned around to see the helicopter collide with a power line guy wire and the ground before bursting in flames. (NTSB Identification: ERA10LA348)

(4) Title 14 Code of Federal Regulations 91.107(a)(3) states that each person "must occupy an approved seat or berth with a safety belt and, if installed, shoulder harness, properly secured about him or her during movement on the surface, takeoff, and landing." (NTSB Identification: CEN10FA324)

(5) Another pilot reported hearing "emergency emergency" transmitted on the control tower frequency shortly after the accident airplane was cleared for takeoff. (NTSB Identification: WPR11FA184)

It is worthy of note that whereas (4) may didactically give lay readers access to technical literature out of their reach but taken for granted in professional circles, (3) segregates the lay audience from the community of experts by means of scare quotes, which act as hedges. They signal inaccuracies or popularisations unnecessary in expert settings.

\subsection{Detail Disclosure}

The disclosure of detail backs up the final verdict just as any of the pieces of evidence analysed in the course of the investigation, and at the same time reinforces the institution's credibility and democratic principles. However, the level of disclosure or granularity may border on the flouting of Grice's quantity and relation maxims. We may find, at times as direct speech quotations, confidential conversation snippets from cabin voice recordings (6) or domestic scenarios, and medical (7), penal (8) and sentimental (9) information that may explain certain behaviours to determine the accident causes. Italics are mine in all instances.

(6) During the brief conversation between the pilot, his son, and the officers, the purpose of the flight and the circumstances of the collision with trees were not discussed. According to his son, the pilot reported, "The darn thing just quit." The pilot subsequently died from his injuries and was not interviewed. (NTSB Identification: ERA11LA326)

(7) Review of the pilot's medical records indicated that he had a history of depression, anxiety, and obstructive sleep apnea. (NTSB Identification: CEN11GA020)

(8) A review of the pilot's background revealed two prior convictions for driving under the influence of alcohol. These were reported to the Federal Aviation Administration (FAA) on the pilot's medical certificate application. (NTSB Identification: CEN10LA275)

(9) The pilot had been experiencing difficulties in his personal life and had joked with a clergyman about 2 days before the accident that he should, "just fly his plane into the ocean." The pilot was subsequently reported missing. (NTSB Identification: ERA12LA578) 


\section{Conclusion}

It is indeed unusual that accident dockets, expected to be concise technical summaries, may contain such a varied (and often emotionally tinged) range of multifunctional persuasive resources. While it might appear that the voice of the NTSB's verdicts (supposedly omniscient and distal) and its knowledge of details clashes with the intimacy and rapport created by plural storytelling and the amusement of in medias res openings, the overall effect turns out to be the opposite. Strikingly, these strategies serve to depict the NTSB as a flexible authority that hosts a number of other co-narrating voices, convergent with its final decisions and instrumental to approaching the audience to the notion and contexts of risk.

\section{References}

Besley, J.C. \& McComas, K.C. (2014). Fairness, public engagement and risk communication. In J. Árvai \& L. Rivers (Eds.), Effective risk communication (pp. 108-123). London: Routledge.

Boholm, A. \& Corvellec, H. (2014). A relational theory of risk: Lessons for risk communication. In J. Árvai \& L. Rivers (Eds.), Effective risk communication (pp. 8-22). London: Routledge.

Cap, P. (2013). Proximization: The pragmatics of symbolic distance

crossing. Amsterdam: John Benjamins.

Harré, R. \& van Langenhove, L. (1999). Positioning Theory: Moral contexts of intentional action. Oxford: Blackwell.

Hewings, A. (2012). Stance and voice in academic discourse across channels. In K. Hyland \& C. Sancho Guinda (Eds.), Stance and Voice in Written Academic Genres (pp. 187-201).Houndmills, Basingstoke (UK): Palgrave Macmillan.

Hyland, K. \& Jiang, F. (2017). Is academic writing becoming more informal? English for Specific Purposes, 45, 40-51.

Van Dijk, T.A. (2016). Discurso y conocimiento. Una aproximación sociocognitiva. Barcelona: Gedisa.

Zhang, Y. \& O'Halloran, K.L. (2014). From popularization to marketization: The hypermodal nucleus in institutional science news. In E. Djonov \& Zhao, S. (Eds.), Critical Multimodal Studies of Popular Discourse (pp. 160-177). New York, NY: Routledge.

Zwickle, A. \& Wilson, R.S. (2014). Construing risk. In J. Árvai \& L. Rivers (Eds.), Effective risk communication (pp. 190-203). London: Routledge. 